\title{
Ab Initio Metadynamics Calculations Reveal Complex Interfacial Effects in Acetic Acid Deprotonation Dynamics
}

Sohag Biswas and Bryan M. Wong*

Department of Chemical \& Environmental Engineering, Materials Science \& Engineering Program, Department of Physics $\&$ Astronomy, and Department of Chemistry, University of California-Riverside, Riverside, California 92521, USA

E-mail: bryan.wong@ucr.edu,Website:http://www.bmwong-group.com

\begin{abstract}
Acid-base reactions play a central role in solution chemistry, with carboxylic acids being particularly important in atmospheric chemical processes. In this work, we harness metadynamics calculations with Born-Oppenheimer molecular dynamics (BOMD) simulations to understand deprotonation dynamics of acetic acid $\left(\mathrm{CH}_{3} \mathrm{COOH}\right)$ in both bulk and air-water interfacial environments. Collective variables are carefully chosen in our well-tempered metadynamics simulations to capture the deprotonation process in various aqueous configurations. Our findings show that the free energy barrier for deprotonation of acetic acid at the air-water interface is lower than in the bulk, in accordance with the available experimental data. Furthermore, our well-tempered metadynamics calculations suggest that the variations in free energy are primarily due to intricate solvation shell effects.
\end{abstract}




\section{Introduction}

Proton transfer dynamics in complex aqueous environments continue to garner immense interest from both experimentalists and theorists. ${ }^{1,2}$ In particular, the air-water interface presents an unusual discontinuity and asymmetry in intermolecular interactions, resulting in different dynamical properties than those observed in a bulk aqueous environment. For example, molecules that reside at the interface encounter vastly different interactions and undergo unique conformations compared those found in the bulk. ${ }^{3}$ This same abrupt change at the interface can enhance reactivity in various chemical processes, such as the oxidation of halide ions by $\mathrm{OH}$ radicals or $\mathrm{O}_{3}$ in the ozone cycle. ${ }^{4}$ As such, the dissociation of acid molecules at the air-water interface provides a microscopic understanding of reactivity at the interface, which can play additional catalytic roles during these dynamical events. ${ }^{5-7}$

Organic acids such as carboxylic $(\mathrm{RCOOH})$ and dicarboxylic acids have attracted growing interest due to their important role in troposphere chemistry. ${ }^{8,9}$ In particular, acetic acid is a normal metabolite of vegetation ${ }^{10}$ and contributes to less volatile organic compounds (VOCs). ${ }^{11}$ Acetic acid is also found in clouds ${ }^{12-14}$ and fog, ${ }^{15}$ which contributes to a significant fraction of observed gas-phase acidity in the atmosphere. ${ }^{8,16}$ Furthermore, the concentration of these carboxylic acids may increase pollution in urban environments and is a substantial contributor to photochemical smog. ${ }^{16}$ Although acetic acid is weaker than other mineral acids such as nitric and sulfuric acid, carboxylic acids are found in much larger concentrations and may have a more substantial overall effect on the free acidity of rainwater. In particular, Knee and Galloway predicted that acetic and formic acid might account for 16 to 35 percent of the free acidity of precipitation in the United States alone. ${ }^{17}$

Recent experimental studies on acetic acid have shown an enhanced acidity on the surface of water, with only a slight dissociation to the carboxylate anion at low concentrations in water. ${ }^{18}$ At bulk concentrations ( $\approx 0.5$-mole fraction of acetic acid), approximately $99 \%$ of the acetic acid remains undissociated. ${ }^{19,20}$ The deprotonation reactions of formic and acetic acid in aqueous environments have also been probed with X-ray photoelectron ${ }^{21-23}$ 
and VSFG spectroscopic techniques. ${ }^{24,25}$ Both techniques reported no or minimal deprotonation at the air-water interface. Previous theoretical calculations, such as micro-hydration techniques, examined binding energies and minimum energy structures of acetic acid-water clusters. ${ }^{26,27}$ Transition path sampling and metadynamics simulations have also been used to predict dissociation mechanisms of acetic acid in bulk ambient water. ${ }^{28,29}$ Finally, additional theoretical studies have also confirmed that carboxylic acids, such as acetic acid, have a particular affinity towards the air-water interface due the presence of both hydrophilic hydroxyl and hydrophobic methyl groups. ${ }^{30,31}$

Motivated by these innovative experimental advancements and recent studies, we decided to further investigate the deprotonation of acetic acid at the air-water interface to provide theoretical insight into these complex dynamical processes. To this end, we have carried out ab initio-based metadynamics simulations to study the deprotonation mechanisms of acetic acid by calculating the free-energy landscape at the air-water interface as well as in a bulk aqueous environment. As such, this work provides mechanistic insight and offers quantum-based predictions of acetic acid in confined aqueous environments for understanding these complex dynamical effects. These computational techniques allow us to (1) probe the detailed mechanisms and time scales of acetic acid deprotonation dynamics and (2) reconstruct the free energy surface in bulk aqueous and air-water interfacial configurations to obtain free energy dissociation barriers in these environments. Based on our findings, we propose underlying mechanistic reasons for the lower free energy barrier of acetic acid deprotonation at the air-water interface compared to the bulk.

\section{Simulations details}

\section{AIMD simulations}

All ab initio molecular dynamics (AIMD) simulations were carried out using the QUICKSTEP ${ }^{32-34}$ module in the CP2K software suite. ${ }^{35,36}$ The QUICKSTEP module in CP2K uti- 
lizes hybrid Gaussian and plane-wave (GPW) ${ }^{37}$ schemes for the efficient calculation of forces and energies. GTH pseudopotentials ${ }^{38,39}$ are used to describe the atomic core electrons, and the Kohn-Sham orbitals for the valence electrons employed the TZV2P basis set. ${ }^{40}$ All of our simulations utilized the BLYP ${ }^{41-43}$ functional with D3 dispersion corrections. ${ }^{44}$ The orbital transformation method with an electronic gradient tolerance value of $1 \times 10^{-5}$ Hartree was used as the convergence criteria for the SCF cycle. ${ }^{32}$ At each molecular dynamics step, the initial guess was given by the stable predictor-corrector extrapolation method. ${ }^{45,46} \mathrm{~A}$ time step of 0.5 fs was used for integrating the equations of motion, and the energy cut-off for the auxiliary plane-wave basis set was fixed to 300 Ry. All simulations were carried out at 300 K using a Nose-Hoover chain thermostat. ${ }^{47,48}$

\section{Bulk simulations}

For the bulk aqueous configuration, we placed a single acetic acid molecule with 64 explicit waters in a cubic box with side lengths of $12.42 \AA$ (which closely approximates the experimental density of 64 water molecules). The initial geometry was generated using the PACKMOL software package, ${ }^{49}$ and a 15 -ps NPT simulation was subsequently carried out at $300 \mathrm{~K}$ to obtain the equilibrium volume for a single acetic acid molecule in water. The pressure was controlled by the barostat suggested by Mundy and co-workers. ${ }^{50}$ The NPT simulation was subsequently used to determine the density of this acetic acid-water mixture. The resulting density of the mixture reached $1.001 \mathrm{~g} / \mathrm{cm}^{3}$, which corresponds to an average box length of $12.12 \AA$. After fixing the average box length, we continued with an additional 5-ps of an NVT simulation with a massive Nose-Hoover chain thermostat. For our production runs, we subsequently performed another 50 ps of an NVT simulation with a global Nose-Hoover thermostat. Periodic boundary conditions were implemented in the $x, y$, and $z$ directions for the bulk aqueous simulations. 
(a)

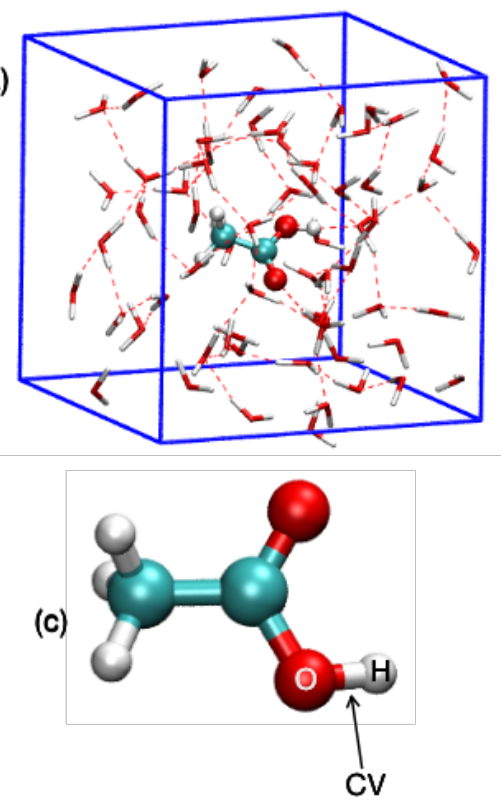

(b)

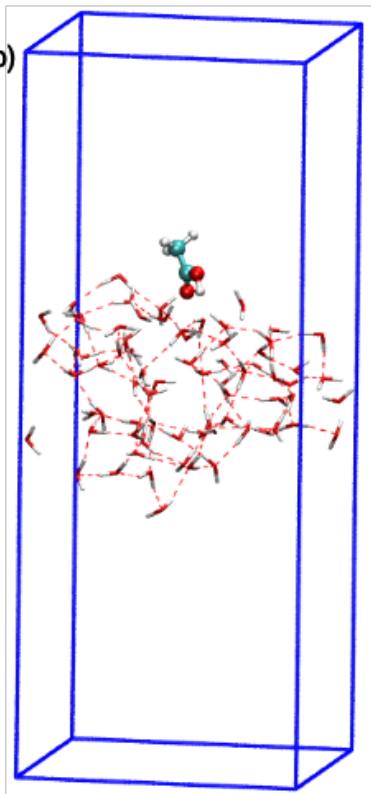

Figure 1: Representative (a) bulk and (b) slab configurations of acetic acid in explicit water. (c) An acetic acid molecule $\left(\mathrm{CH}_{3} \mathrm{COOH}\right)$ depicted with the collective variable $(\mathrm{CV})$ used in this work.

\section{Interfacial simulations}

For our interfacial acetic acid-water model, we generated a thin water slab comprised of 73 water molecules within a rectangular supercell with dimensions of $15.56 \AA \times 15.56 \AA \times 40$ $\AA$, as shown in Figure 1b. For understanding surface effects, slab models with very similar compositions have been previously shown to produce a consistent air-water interface. ${ }^{31,51,52}$ For the air-water interface configuration, periodic boundary conditions were applied in the $x$ and $y$ directions of the slab. Thus, the $x y$-plane of the slab system remains parallel to the surface, and the $z$-axis forms the surface normal where acetic acid collides with the surface water molecules. Before placing an acetic acid molecule in the slab, the bare water slab was equilibrated with an NVT ensemble for 15 ps by applying a massive Nose-Hoover chain thermostat at $300 \mathrm{~K}$. After equilibration, a single acetic acid molecule was placed 6 $\AA$ above the center of mass of the slab along the $z$-direction. The center of mass of the acetic acid molecule was constrained, and an additional 2 ps NVT equilibration was carried out with the massive Nose-Hoover chain thermostat. Next, the constraint was released, and 
an additional 50-ps NVT simulation was carried out. We also carried out calculations at a slightly higher temperature of $330 \mathrm{~K}$ to probe structural and dynamical effects of acetic acid at the air-water interface. As suggested by previous studies, ${ }^{31,53,54}$ we used a harmonic constraint with a constant spring value of 1 Hartree throughout the simulations to maintain the slab at the origin of the coordinate system.

\section{Metadynamics simulations}

An enhanced sampling method was required to explore the deprotonation mechanisms of aqueous acetic acid and obtain the free energy barrier of dissociation. In metadynamics simulations, the free energy surface is produced from a biasing potential, which depends on the set of predefined collective variables. The initial structure for our metadynamics simulations was extracted from pre-equilibrated BOMD simulations. To determine the free energy surface of activation for the dissociation of acetic acid in the bulk vs. the air-water interface between its neutral form and anionic form, a minimum free energy path was calculated by adequately defining the collective variable. The collective variable describes the coordination number during the metadynamics simulations, and the following expression was used to calculate the value of the coordination number $(\mathrm{CN})$ :

$$
\mathrm{CV}_{1} \text { or } \mathrm{CN}=\frac{1-\left(\frac{d_{\mathrm{AB}}}{d_{0}}\right)^{p}}{1-\left(\frac{d_{\mathrm{AB}}}{d_{0}}\right)^{p+q}}
$$

where $d_{\mathrm{AB}}$ is the distance between atoms $\mathrm{A}$ and $\mathrm{B}$, and $d_{0}$ is the reference distance or fixed cut-off parameter. This parameter distinguishes the standard bond distance between atoms $\mathrm{A}$ and $\mathrm{B}$. In the present case, we chose the distance between the $\mathrm{O}$ and $\mathrm{H}$ atoms of the hydroxyl group of $\mathrm{CH}_{3} \mathrm{COOH}$ as a collective variable. In equation $1, p$ and $q$ are constants, which were set to $p=q=6$. These values are utilized to differentiate between the coordinated and non-coordinated states. For the $\mathrm{O}-\mathrm{H}$ pair of atoms, the $\mathrm{CN}$ value is almost unity (0.8 in practice) when $d_{\mathrm{AB}}<d_{0}$, and the value of $\mathrm{CN}$ approaches zero rapidly $(0.26$ 
in practice) when $d_{\mathrm{AB}}>d_{0}$. In the preesent case, the $d_{0}$ value for O-H was set to $1.60 \AA$. The deposition rate for the Gaussian hills was set to 100 steps during the metadynamics simulations, and the Gaussian width and height were set to 0.1 and 0.0005 Hartree, respectively. We carried out two metadynamics calculations using different initial conditions for the bulk aqueous and interfacial environments. The metadynamics simulations were run for 22 ps (trajectory 01) and 36 ps (trajectory 02) for the bulk aqueous and 8 ps (trajectory 01) and 10 ps (trajectory 02) for air-water configurations, respectively. We confirmed a good sampling and convergence of the reactant, product, transition state, and free energy differences, particularly for the deprotonation barrier. It is worth mentioning that more complex metadynamics approaches (with as many as three collective variables) have been used to explore high-dimensional configurations. ${ }^{28}$ However, these approaches may take a considerable amount of computational time, even when well-tempered metadynamics simulations are used. As such, the single-CV approach used in our calculations allows convergence of the free energy profile in a reasonable amount of time and computational effort.
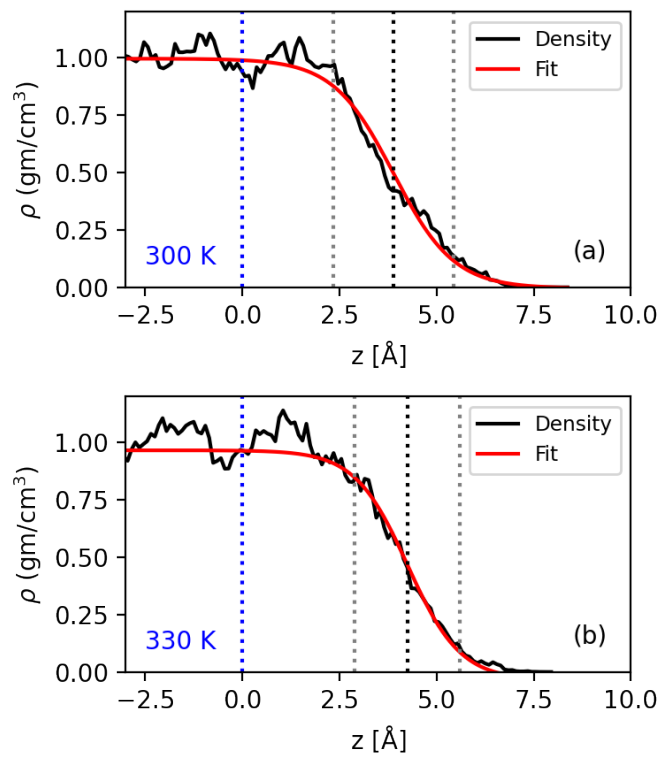

Figure 2: (a) Density profile of water slab at (a) $300 \mathrm{~K}$ and (b) $330 \mathrm{~K}$. The dotted blue lines in both panels indicate the center of the slab, set to zero in our calculations The vertical black dotted lines in both panels represent $z_{\text {GDS }}$, and grey dotted lines indicate the interfacial thickness, $z_{\mathrm{GDS}} \pm \delta$. 


\section{Results and discussion}

\section{Density profile of interfacial water}

The density of bulk water can be estimated by fitting the density profile in the water slab configurations. We calculated the density profile of water along the surface normal ( $z$ axis) and fitted it with a hyperbolic tangent function:

$$
\rho(z)=\frac{1}{2}\left(\rho_{1}+\rho_{v}\right)-\frac{1}{2}\left(\rho_{1}-\rho_{v}\right) \tanh \left(\frac{z-z_{\mathrm{GDS}}}{\delta}\right)
$$

where $\rho_{1}$ represents the density of bulk water, and $\rho_{v}$ is the density of the water vapor phase. The $\rho_{v}$ value was set to zero during the density profile fitting. In the above equation, $z_{\text {GDS }}$ is the $z$-coordinate of the Gibbs dividing surface (the point where the density is half of the bulk water density), and $\delta$ is the thickness parameter of the interface. The density profiles of water from the acetic acid-water slab systems at 300 and $330 \mathrm{~K}$ are shown in Figure 2. Fitting the $300 \mathrm{~K}$ density profile to equation 2 gives a water density $\left(\rho_{1}\right)$, Gibbs dividing surface $\left(z_{\mathrm{GDS}}\right)$, and interfacial thickness $(\delta)$ of $0.99 \mathrm{gm} / \mathrm{cm}^{3}, 3.90 \AA$, and $1.55 \AA$, respectively. For $330 \mathrm{~K}$, the density fitting procedure gives $\rho_{1}, z_{\mathrm{GDS}}$, and $\delta$ values of $0.93 \mathrm{~g} / \mathrm{cm}^{3}, 4.25 \AA$, and $1.35 \AA$, respectively. The computed density profile at $300 \mathrm{~K}$ is very similar to the density profile obtained by Mundy and co-workers, which used a gradient-corrected BLYP functional. ${ }^{55}$ Our calculations of the water density $\left(=0.99 \mathrm{gm} / \mathrm{cm}^{3}\right)$ at $300 \mathrm{~K}$ match closely with previous studies that reported densities of $1.06 \mathrm{gm} / \mathrm{cm}^{3}$ and $1.07 \mathrm{gm} / \mathrm{cm}^{3}$ at $295 \mathrm{~K}{ }^{56,57}$

\section{Surface affinity of acetic acid and hydroxyl bond length}

Figure 3 shows the dynamics of acetic acid within a water slab at 300 and $330 \mathrm{~K}$. The gray stripe indicates the top surface of the water slab where acetic acid will collide/reside. We define the thickness of the interface to be $z_{\mathrm{GDS}}-\delta<z<z_{\mathrm{GDS}}+\delta$ within the water slab for all temperatures. We did not observe any acetic acid scattering from the slab 

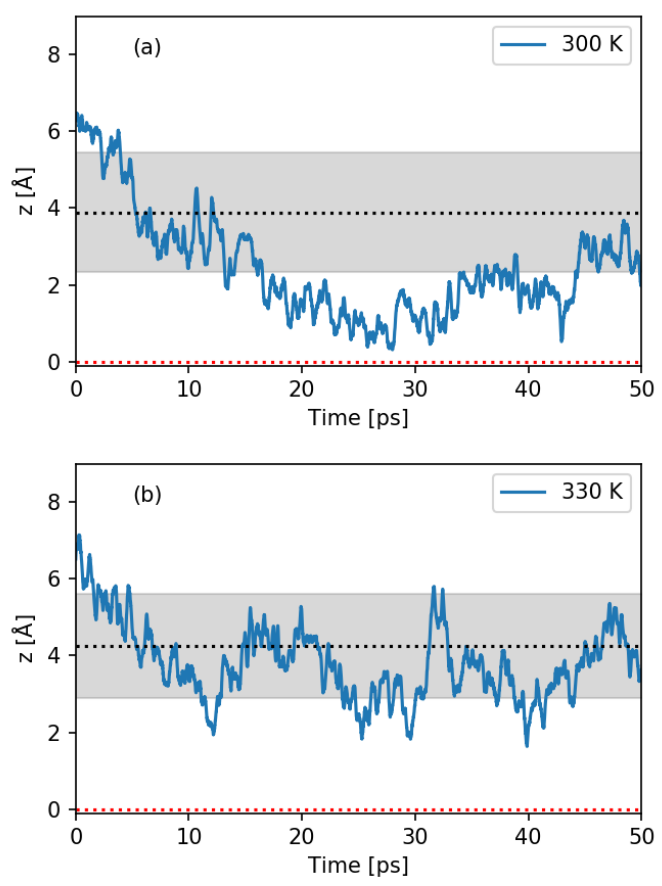

Figure 3: Vertical position of the carbon atom in the $-\mathrm{COOH}$ group of acetic acid relative to the center of the slab. Panels (a) and (b) show simulations carried out at 300 and $330 \mathrm{~K}$, respectively. The black dotted line in both panels denotes the position of the Gibbs dividing surface. The gray stripe indicates the thickness $( \pm \delta)$ of the slab in both panels. The red dotted line in panels (a) and (b) denote the center of the slab.

interface or diffusion into the bulk. Due to restrictions associated with the computationally intensive ab initio simulations, a 50 ps trajectory length is likely too short to observe acetic acid diffusion into the bulk. As such, the acetic acid molecule remains essentially near the air-water interface during the simulations. Figure S1 in the Supplementary Material plots the acetic acid hydroxyl bond length as a function of time at 300 and $330 \mathrm{~K}$ for the interfacial simulations. The horizontal blue dotted line at $1.00 \AA$ indicates the equilibrium $\mathrm{O}-\mathrm{H}$ bond distance. During the AIMD simulations, the $\mathrm{O}-\mathrm{H}$ bond is stretched from its equilibrium position due to hydrogen-bond interactions with neighboring water molecules, but no trajectories resulted in acetic acid deprotonation at the surface. 

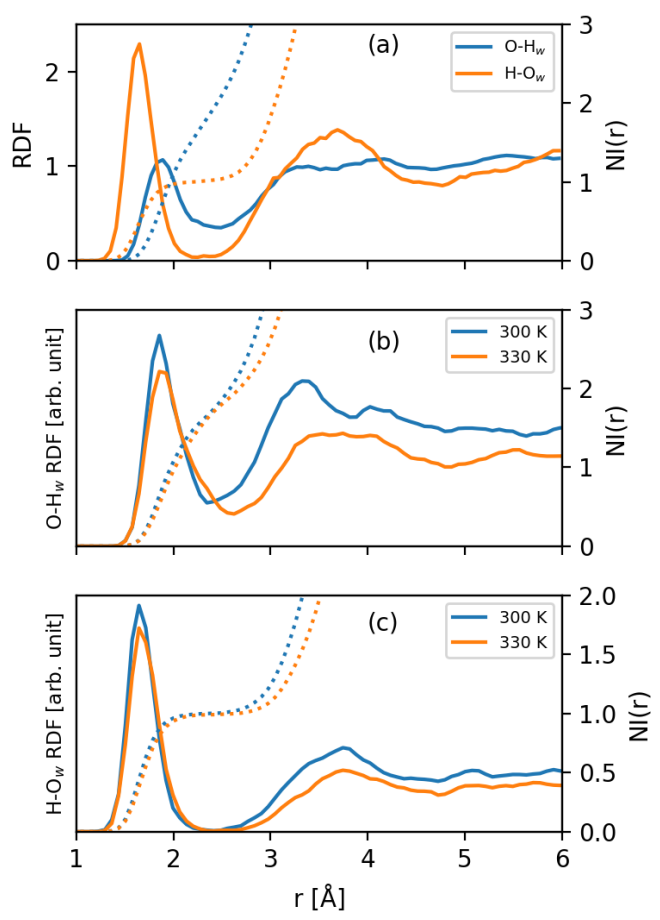

Figure 4: (a) Radial distribution functions (RDFs) for $\mathrm{O}-\mathrm{H}_{w}$ and $\mathrm{H}-\mathrm{O}_{w}$ pairs in the bulk aqueous environment. (b,c) RDFs for the $\mathrm{O}-\mathrm{H}_{w}$ and $\mathrm{H}-\mathrm{O}_{w}$ pairs in a slab configuration at 300 and $330 \mathrm{~K}$. O and $\mathrm{H}$ represents the carbonyl oxygen and hydroxyl hydrogen atom of the - COOH group of acetic acid. The solid line indicates the RDF in all panels, and the dashed line denotes the number integral.

\section{Solvation shell structure}

We examined the structural correlations of acetic acid in the bulk aqueous environment and air-water interface by calculating radial distribution functions (RDFs). The calculated RDFs of $\mathrm{O}-\mathrm{H}_{w}$ and $\mathrm{H}-\mathrm{O}_{w}$ pairs for bulk vs. slab configurations are shown in Figure 4. The O and $\mathrm{H}$ atoms represent the carbonyl oxygen and the hydroxyl hydrogen atom of acetic acid, respectively. The $\mathrm{O}-\mathrm{H}_{w}$ and $\mathrm{H}-\mathrm{O}_{w}$ RDFs for a bulk aqueous environment are shown in Figure 4(a). The number integral represents the coordination number. The first peak of the $\mathrm{O}-\mathrm{H}_{w}$ profile at the bulk aqueous environment occurs at $1.90 \AA$, followed by a minimum at $2.50 \AA$. The integration of the peak up to the first minimum position leads to a hydration number of 2.01. This peak is correlated with the hydrogen bond formation between the carbonyl oxygen of acetic acid and the $\mathrm{H}$ atom of the surrounding water molecules. Therefore, the 
carbonyl oxygen atom of acetic acid participates in approximately two hydrogen bonds in a bulk aqueous environment. The $\mathrm{H}-\mathrm{O}_{w}$ RDF pair has a sharp first peak at $1.65 \AA$, followed by a minimum at $2.32 \AA$ in the bulk aqueous environment. The height of the peak in the $\mathrm{H}-\mathrm{O}_{w}$ $\mathrm{RDF}$ is somewhat larger compared to the $\mathrm{O}-\mathrm{H}_{w}$ RDF pair in the bulk. The sharp peak for the $\mathrm{H}-\mathrm{O}_{w}$ pair also indicates a strong hydrogen bond between the hydroxyl hydrogen of the acetic acid molecule and the oxygen atom of the surrounding water molecules. The value of the number integral up to the first minimum of the $\mathrm{H}-\mathrm{O}_{w} \mathrm{RDF}$ is 1.0, which corresponds to the hydrogen bond number. Our reported values of acetic acid-water RDFs in bulk water agree with earlier simulation studies. ${ }^{58}$

The $\mathrm{O}-\mathrm{H}_{w}$ and $\mathrm{H}-\mathrm{O}_{w}$ RDFs at the air-water interface are displayed in Figures 4(b) and 4(c), respectively, for all the temperatures. The RDF units at the interface are arbitrary since the box lengths in the $x, y$, and $z$ directions are different. Therefore, a relative unit of measurement is used to calculate the relative atom distribution vs. the distance between various $\mathrm{RDF}$ pairs at the air-water interface. The first peak of the $\mathrm{O}-\mathrm{H}_{w} \mathrm{RDF}$ profiles occurs at $1.86 \AA$ for all of the temperatures. The position of the peak does not change appreciably with temperature; however, an increase in temperature does decrease the peak height. At $300 \mathrm{~K}$, the first minimum of the $\mathrm{O}-\mathrm{H}_{w} \mathrm{RDF}$ is located at $2.35 \AA$; in contrast, the position of this minimum becomes localized at $2.63 \AA$ when the temperature is increased to $330 \mathrm{~K}$. The integration of the peaks up to the first minimum contributes a $1.63(300 \mathrm{~K})$ and 1.90 (330 K) hydrogen bond coordination for the carbonyl oxygen atom of acetic acid at the air-water interface. The hydrogen bond coordination number for $\mathrm{O}-\mathrm{H}_{w}$ pairs at the interface is slightly lower than the bulk aqueous environment.

Sharp peaks occur at $1.65 \AA$ and are accompanied by successive minima at $2.50 \AA$ for $\mathrm{H}-\mathrm{O}_{w}$ RDF pairs at the surface. For the $\mathrm{H}-\mathrm{O}_{w}$ RDF pairs, the peak and minima positions are unchanged when the temperature is varied. The integration of these peaks up to the first minima positions leads to a hydration number of 1.0. The sharp peak and deeper minima of the $\mathrm{H}-\mathrm{O}_{w}$ RDF pair in the bulk aqueous and air-water configurations suggest that acetic acid 
makes significant hydrogen bonding interactions with the hydroxyl group compared to the $\mathrm{C}=\mathrm{O}$ group. The hydroxyl oxygen of the acetic acid can also act as a proton acceptor to form a hydrogen bond with neighboring water molecules. To investigate these dynamical effects, we also calculated $\mathrm{O}_{\text {hydroxyl }}-\mathrm{H}_{w}$ RDFs in Figure S2 within the Supplementary Material. The RDFs show tiny peaks between 1.80 to $2.00 \AA$ for the bulk aqueous environment and peaks at 2.00 to $2.50 \AA$ for the air-water interface, indicating direct interaction of water molecules with the hydroxyl oxygen atom. However, this interaction cannot be directly classified as a hydrogen-bond interaction, since the probability of observing these structures during the simulations was negligible.

\section{Hydrogen bond dynamics and solvent orientation}
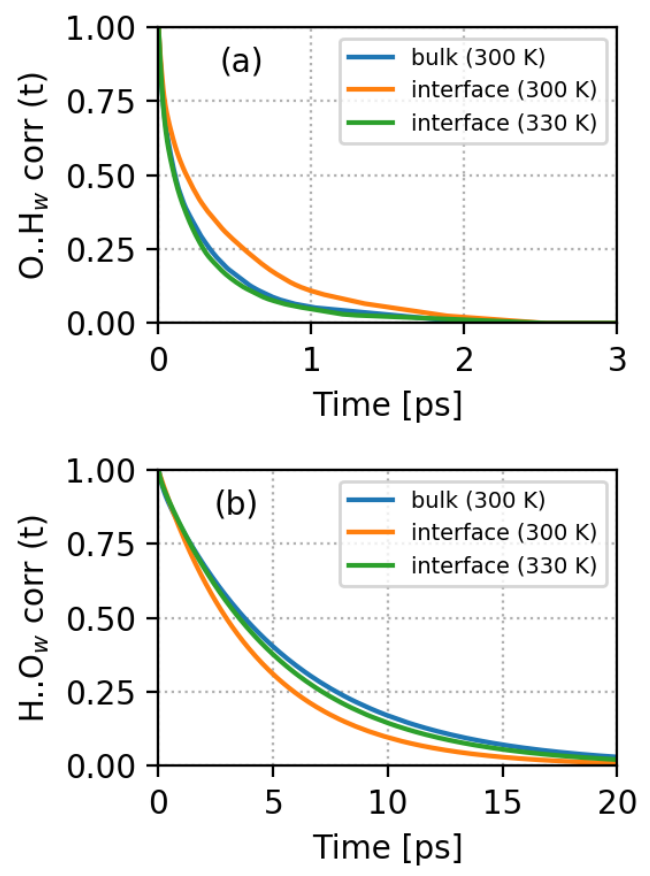

Figure 5: Panel (a) shows the continuous hydrogen bond auto-correlation functions for $\mathrm{O} \cdot \mathrm{H}_{w}$ pairs in the bulk aqueous and air-water interface environments. Panel (b) shows the continuous hydrogen bond auto-correlation functions for $\mathrm{H} \cdots \mathrm{O}_{w}$ pair in the bulk aqueous and air-water interface environments.

The acetic acid molecule contains carbonyl $(-\mathrm{C}=\mathrm{O})$ and hydroxyl $(-\mathrm{OH})$ groups, both of 
which can form hydrogen bonds with neighboring water molecules in the bulk and interfacial configurations. To gain a deeper understanding of the strength and dynamic stability of these hydrogen bonds, we calculated a continuous hydrogen bond auto-correlation function using a population correlation function approach. ${ }^{59-62}$ The continuous hydrogen bond autocorrelation function describes the probability that a selective hydrogen-bonded pair continues to be hydrogen-bonded up to a time $t$, which is defined by the following equation:

$$
S_{\mathrm{HB}}(t)=\left\langle\frac{h_{i j}(0) H_{i j}(t)}{h_{i j}(0)^{2}}\right\rangle .
$$

The variables $h_{i j}$ and $H_{i j}$ are the hydrogen bond population operators that account for the distance between $i$ and $j$ pairs. The bracket indicates an average of all types of hydrogen bond pairs. The operator, $h_{i j}$, is unity when a particular type of hydrogen bonding pair is hydrogen-bonded at time $t$ and zero otherwise. Likewise, $H_{i j}$ is equal to 1 if a hydrogen bond continuously exists from time 0 to $t$; otherwise, it is zero. The lifetime, $\tau_{\mathrm{HB}}$, associated with the hydrogen bond auto-correlation function can be calculated via the integration of equation 3 :

$$
\tau_{\mathrm{HB}}=\int_{0}^{\infty} S_{\mathrm{HB}}(t) d t .
$$

In the present case, we calculated continuous hydrogen bond auto-correlation functions for $\mathrm{O} \cdots \mathrm{H}_{w}$ and $\mathrm{H} \cdots \mathrm{O}_{w}$ pairs, which are shown in Figure 5. Based on bulk-water simulations, Luzar and Chandler ${ }^{59-61}$ developed geometric criteria for designating hydrogen bonding such that the O-O distance should be $\leq 3.35 \AA$, and the corresponding hydrogen bond cut-off angle is $30^{\circ}$. We used a cut-off angle of $45^{\circ}$ to designate an acetic acid-water hydrogen bond in the current work. In other studies, a cut-off angle of $30^{\circ}$ was used, but a less stringent cut-off angle of $45^{\circ}$ can also be utilized for water-solute hydrogen bond interactions.

Our calculated results reveal that the $\mathrm{O} \cdots \mathrm{H}_{w}$ hydrogen-bond auto-correlation function (Figure 5a) decays rapidly compared to that of $\mathrm{H} \cdots \mathrm{O}_{w}$ (Figure $5 \mathrm{~b}$ ). The $\mathrm{O} \cdots \mathrm{H}_{w}$ hydrogen bond correlation functions converged to zero within $2 \mathrm{ps}$, while the $\mathrm{H} \cdots \mathrm{O}_{w}$ correlation func- 
tions converged to zero within 20 ps. The faster decay of the $\mathrm{O} \cdots \mathrm{H}_{w}$ hydrogen bond pair indicates that acetic acid forms weaker hydrogen bonds with neighboring water molecules via the carbonyl moiety. We fitted the correlation decays to a single exponential function to obtain respective lifetime values of the hydrogen bond pairs, which are shown in Table 1. The lifetimes for the $\mathrm{O} \cdot \mathrm{H}_{w}$ pairs in the bulk aqueous and air-water interface configurations are similar. In addition, when the temperature is increased from 300 to $330 \mathrm{~K}$, the lifetime of the $\mathrm{O} \cdot \mathrm{H}_{w}$ pair slightly decreases. The longer lifetime of the $\mathrm{H} \cdots \mathrm{O}_{w}$ pair in the bulk aqueous environment at $300 \mathrm{~K}$ compared to the air-water interface indicates a more stable hydrogen bond formation in the bulk environment. In addition, we also observed that the lifetime of the $\mathrm{H} \cdots \mathrm{O}_{w}$ pair at the interface increases with temperature. Overall, the lifetime of the $\mathrm{H} \cdots \mathrm{O}_{w}$ pair is long compared to the $\mathrm{O} \cdot \cdot \mathrm{H}_{w}$ pair in both the bulk aqueous and airwater interface environments. We also calculated the time-dependent fluctuations of various acetic acid-water hydrogen bonds with the aforementioned hydrogen-bond criteria. Figure S3 in the Supplementary Material shows the time-dependent fluctuations of the hydrogen bonds. The average number of hydrogen bonds are found to be 1.01 and 1.75 for the $\mathrm{H} \cdots \mathrm{O}_{w}$ and $\mathrm{O} \cdot \mathrm{H}_{w}$ pairs, respectively, in the bulk aqueous environment. The average number of H-bonds is 0.99 at both temperatures for the $\mathrm{H} \cdots \mathrm{O}_{w}$ pairs at the air-water interface. Simultaneously, the $\mathrm{O} \cdot \mathrm{H}_{w}$ pair forms $0.91(300 \mathrm{~K})$ and $1.37(330 \mathrm{~K})$ hydrogen bonds at the air-water interface.

Table 1: Hydrogen bond lifetimes $\left(\tau_{\mathrm{HB}}\right)$ in ps for the various acetic acid-water hydrogen bond pairs.

\begin{tabular}{|c|c|c|}
\hline System & $\tau_{\mathrm{HB}} \mathrm{O} \cdots \mathrm{H}_{w}(\mathrm{ps})$ & $\tau_{\mathrm{HB}} \mathrm{H} \cdots \mathrm{O}_{w}(\mathrm{ps})$ \\
\hline Bulk $(300 \mathrm{~K})$ & 0.42 & 5.79 \\
\hline Air-water interface $(300 \mathrm{~K})$ & 0.56 & 4.28 \\
\hline Air-water interface $(330 \mathrm{~K})$ & 0.40 & 5.24 \\
\hline
\end{tabular}

The hydrogen bond auto-correlation calculations suggest that the $\mathrm{H} \cdots \mathrm{O}_{w}$ hydrogen bond pair forms a stronger hydrogen bond than the $\mathrm{O} \cdot \mathrm{H}_{w}$ pair in the bulk aqueous environment and at the air-water interface. The $\mathrm{O} \cdot \mathrm{H}_{w}$ pair also forms a more robust hydrogen bond 


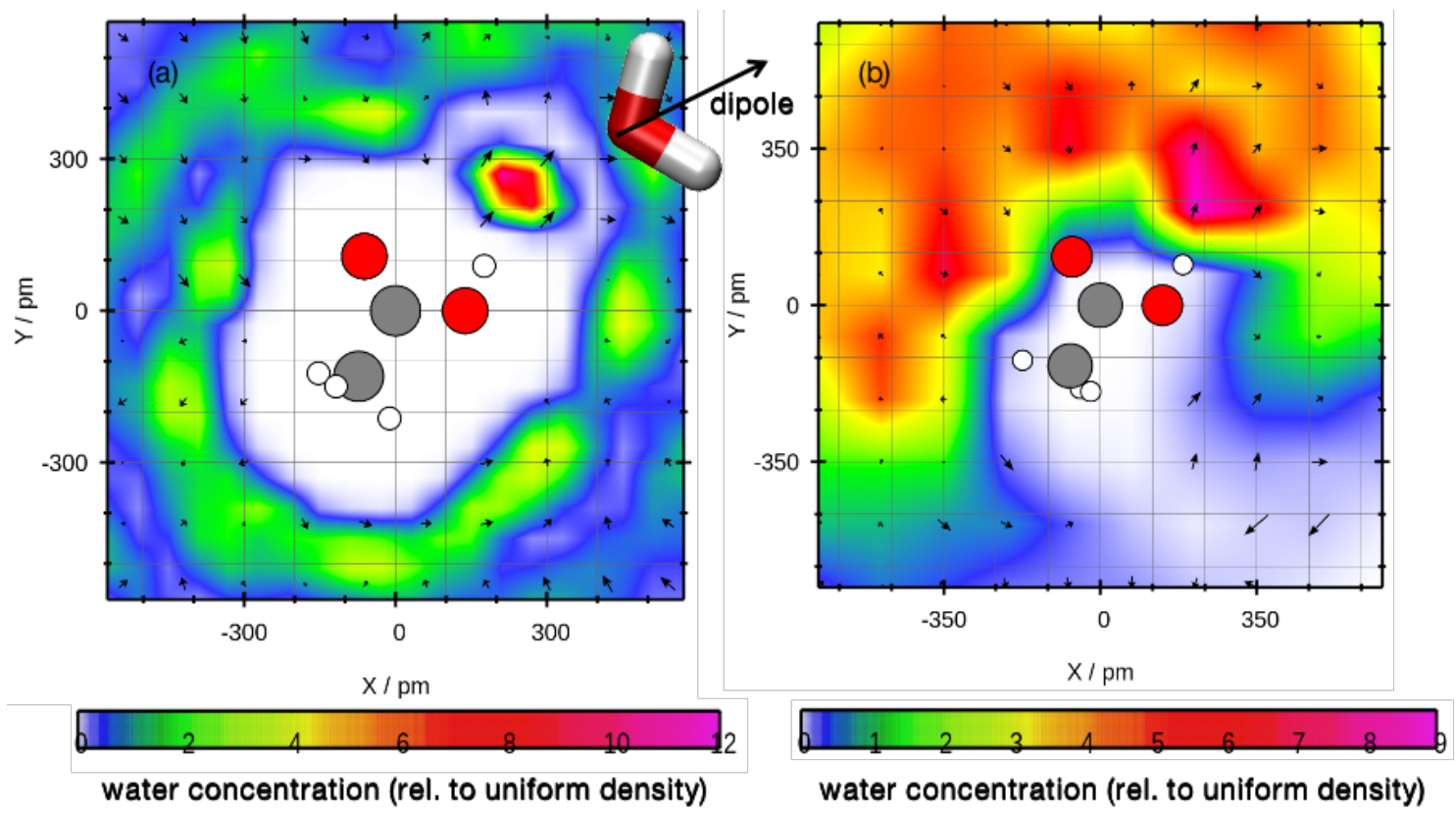

Figure 6: Average particle density of water molecules near an acetic acid molecule superimposed on the average orientation of dipole vectors (arrows) of water molecules. Panels (a) and (b) represent the bulk and interfacial systems, respectively. Gray, red, and white circles denote the carbon, oxygen, and hydrogen atoms, respectively. The carbon atom of the $-\mathrm{COOH}$ group is fixed at the center throughout the calculations.

in the bulk aqueous environment compared to the air-water interface. To understand this, we performed a plane projection analysis using the TRAVIS software package ${ }^{63,64}$ shown in Figure 6. In these plane projection calculations, the acetic acid in the bulk aqueous environment and at the air-water interface is fixed. The color scales in Figures 6a and 6b designate the average particle density of water molecules at the given position relative to the uniform density. A value greater than one implies that water can be found at that location compared to some arbitrary position, whereas a value less than one denotes depletion of water molecules at that position. The vectors in Figure 6 describe the average orientation of the dipole vector of the water molecules. From Figures $6 \mathrm{a}$ and $6 \mathrm{~b}$, we see that the particle density of water molecules near the hydroxyl group is higher compared to the $\mathrm{C}=\mathrm{O}$ group, with the vectors pointing away from this group. In contrast, there is no clear preferential orientation of the dipole vectors of the water near the $\mathrm{C}=\mathrm{O}$ group of the acetic acid molecule (in both the bulk and interfacial environments), resulting in very small average vectors. 
Due to this orientation of water molecules, the $\mathrm{H} \cdots \mathrm{O}_{w}$ hydrogen bond is stronger than the $\mathrm{O} \cdot \mathrm{H}_{w}$ acetic acid-water hydrogen bonds. Comparing the orientation of water molecules around the hydroxyl group in the bulk aqueous environment and at the air-water interface, we find that the particle density of water molecules is higher in the bulk configuration, indicating a stronger $\mathrm{H} \cdots \mathrm{O}_{w}$ hydrogen bond. For the air-water interface simulations, we observe a slightly higher hydrogen-bond coordination number in conjunction with a lower hydrogen-bond lifetime for the $\mathrm{O} \cdot \mathrm{H}_{w}$ pair at $330 \mathrm{~K}$ (compared to $300 \mathrm{~K}$ ). To understand this counter-intuitive behavior, we calculated the combined distribution function (CDF) at 300 and 330 K in Figures S4 and S5 within the Supplementary Material. In the various CDF contour plots, the $x$-axis denotes the $\mathrm{O} \cdot \mathrm{H}_{w} \mathrm{RDF}$, and the $y$-axis represents the hydrogenbond cut-off angle distribution. At $330 \mathrm{~K}$, the hydrogen-bond cut-off angle distribution is slightly wider but has a lower intensity than the configuration at $300 \mathrm{~K}$. In addition, the first minimum of the $\mathrm{O} \cdots \mathrm{H}_{w}$ RDF is somewhat shifted towards a larger distance at $330 \mathrm{~K}$. As such, even though the $\mathrm{O} \cdot \mathrm{H}_{w}$ RDF has a slightly higher coordination number, the longer $\mathrm{O} \cdot \mathrm{H}_{w}$ distance and wider hydrogen-bond cut-off angle make it marginally weaker at 330 K.

\section{Free energy profile}

In this section, we further analyze the free energy profile of acetic acid in a bulk aqueous environment and at the air-water interface. To enable a critical assessment of acetic acid deprotonation dynamics, longer simulations as well as a precise selection of collective variables is essential. The well-tempered metadynamics simulations in the present study were carried out by acquiring a single collective variable (CV), denoted as CV1, which is the coordination number from $\mathrm{O}$ to $\mathrm{H}$, as shown in Figure 1c.

Figure 7 depicts the free energy profile for acetic acid deprotonation in a bulk aqueous environment from trajectory 01, which was generated by well-tempered metadynamics simulations. Initially, CV1 fluctuates near $\approx 0.90 \AA$, and after 5.45 ps, the system departs from 

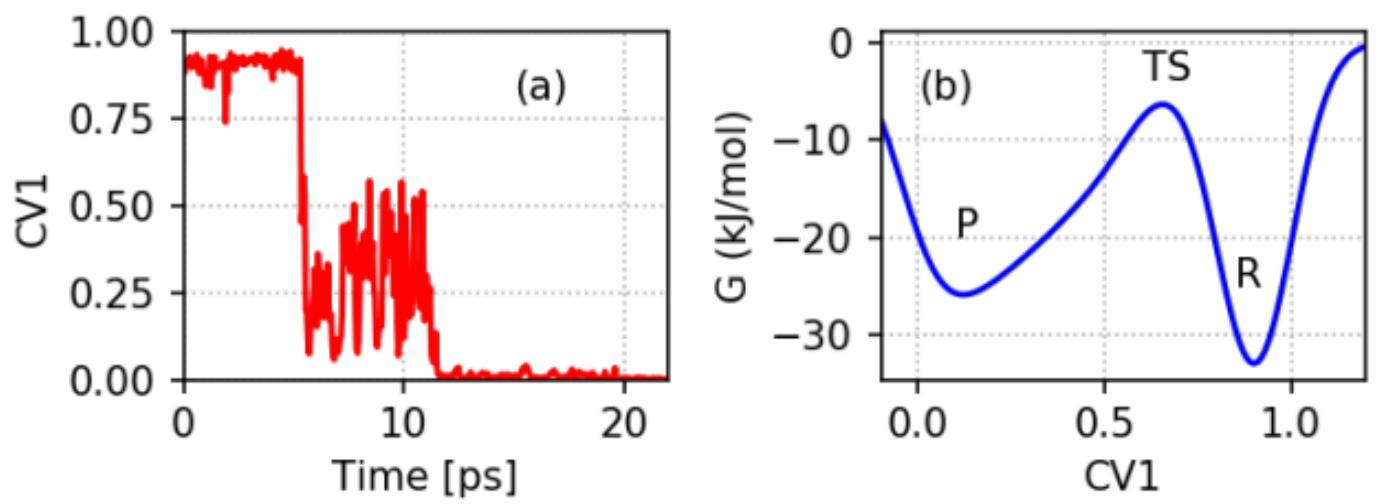

(c)
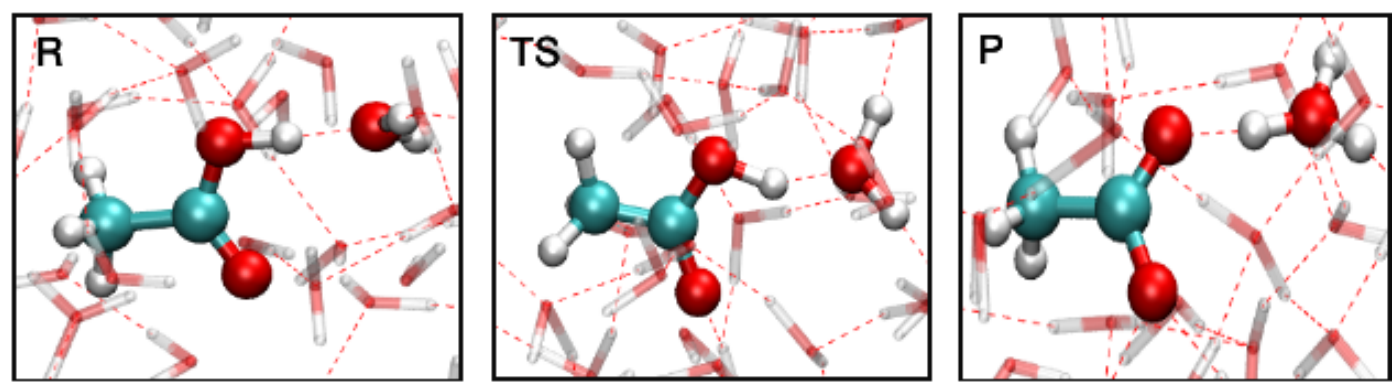

Figure 7: (a) Fluctuations in CV1 obtained from well-tempered metadynamics simulations as a function of time in the bulk aqueous environment. (b) Free energy landscape of acetic acid deprotonation in the bulk aqueous environment calculated from well-tempered metadynamics simulations. (c) Reactant (R), transition state (TS), and product (P) obtained from the welltempered metadynamics simulations.

the reactant well to the product well, as observed by the change in CV1 values. After this point, CV1 changes from 0.9 to 0.1 and fluctuates between 0.1 to 0.5 angstroms. After $11 \mathrm{ps,}$ CV1 fluctuates around 0 (i.e., the O-H bond of acetic acid breaks). The reactant well (R) is positioned at $\approx 0.90$ (see Figure $7 \mathrm{~b}$ ), which indicates an intact acetic acid molecule that is hydrogen-bonded to neighboring water molecules. The free energy value corresponding to the $\mathrm{R}$ state is $-32.95 \mathrm{~kJ} / \mathrm{mol}$ which manifests itself as a deep single-centered well. The dissociated acetic acid product, $\mathrm{P}$, is defined by $\mathrm{CV} 1 \approx 0.1$, and the transition state (TS) is located between the $\mathrm{R}$ and $\mathrm{P}$ states. The corresponding $\mathrm{CV} 1$ value for the transition state is $\approx 0.65$. The free energy value for the TS is $-6.55 \mathrm{~kJ} / \mathrm{mol}$, which corresponds to a free energy activation barrier of $26.4 \mathrm{~kJ} / \mathrm{mol}$ for acetic acid deprotonation in the bulk aqueous environment. The reactant, transition state, and product for the acetic acid deprotonation 
in the bulk aqueous environment are shown in Figure 7c. In the Supplementary Material, we present an additional free energy profile (Figure S6) from trajectory 02 that utilizes different initial conditions in the bulk aqueous environment. Figure S6(a) represents fluctuations of CV1 values, and Figure S6(b) shows the free energy profile. In this case, the reactant proceeds towards the product state via a stable transition state with a free energy barrier value of $29.65 \mathrm{~kJ} / \mathrm{mol}$. A tiny minimum (corresponding to the contact ion pair) is also observed between the reactant and transition state. The free energy path is very similar to the earlier study obtained by Park et al. ${ }^{28}$ which uses a more complex metadynamics approach with three collective variables. As such, the average free energy value from these two independent trajectories is $28.025 \mathrm{~kJ} / \mathrm{mol}$. Our calculated free energy activation barrier is very close to the CPMD metadynamics result obtained by Tummanapelli et al. for acetic acid deprotonation in a bulk aqueous environment. ${ }^{65}$ However, we note an apparent discrepancy between our result and the one obtained by Tummanapelli et al. for the free energy difference between the product and reactant, which deserves further explanation. Specifically, the free energy profile by Tummanapelli et al. was obtained from a short metadynamics trajectory of $5.2 \mathrm{ps,}$ which actually compares well with our unconverged, intermediate free energy profile obtained after 6.75 ps (cf. Figure S7 in the Supplementary Material). Moreover, Daub et al. ${ }^{66}$ recently criticized the results by Tummanapelli et al. due to their short metadynamics trajectory in conjunction with additional issues with their simulations. ${ }^{65}$ As such, the unconverged free energy profile obtained from the short metadynamics simulations by Tummanapelli et al. is unreliable compared to our long-time metadynamics results (cf. convergence plots as a function of simulation time in Figure S8 of the Supplementary Material).

Compared to other similar systems, our calculated free energy barriers are somewhat higher, such as the $14.8 \pm 0.8,{ }^{66} 14.8,{ }^{31}$ and $17.2 \mathrm{~kJ} / \mathrm{mol}^{67}$ activation barriers for formic acid deprotonation in bulk water. The observed high activation barrier for acetic acid vs. formic acid deprotonation in the bulk is due to the inductive effect of the methyl group in acetic acid. ${ }^{65}$ We have also calculated various distances between the carboxylate oxygen 
and neighboring hydrogen atoms from our metadynamics simulations of the bulk aqueous environment, shown in Figure S9 in the Supplementary Material. The calculations suggest that the neighboring hydrogen atoms remain hydrogen-bonded to these carboxylate oxygen atoms, and no re-protonation occurred during these simulations. To assess the convergence of the metadynamics simulations in the bulk aqueous environment, we show the gradual build-up of the free energy profile as a function of the CV (Figure S7 in the Supplementary Material) and calculate the free energy difference between the transition state and reactant as a function of time (Figure S8 in the Supplementary Material). This analysis, along with the fluctuation of CV values, suggests that our metadynamics simulations are converged.
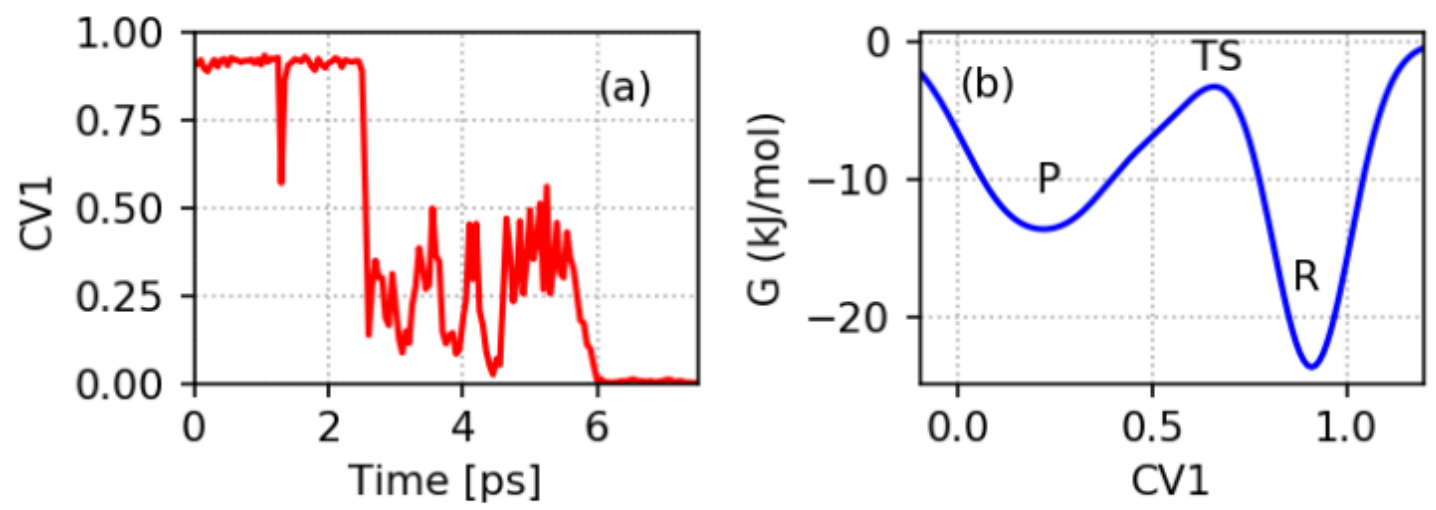

(c)
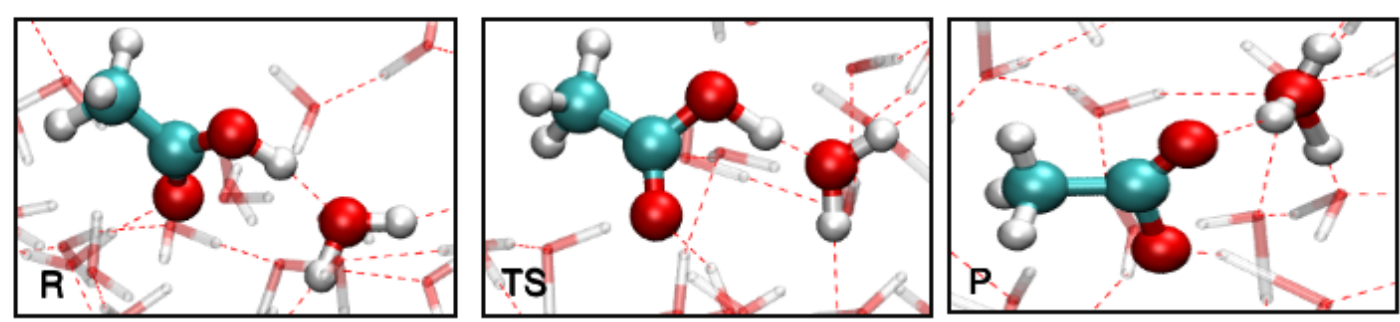

Figure 8: (a) Fluctuations in CV1 obtained from well-tempered metadynamics simulations as a function of time at the air-water interface. (b) Free energy landscape of acetic acid deprotonation at the aqueous interface is calculated from well-tempered metadynamics simulations. (c) Reactant (R), transition state (TS), and product (P) obtained from the well-tempered metadynamics simulations.

The reconstructed free energy surface for the deprotonation of acetic acid at the air-water interface from the trajectory 01 is shown in Figure 8. The initial geometry for the welltempered metadynamics at the air-water interface was obtained from the NVT simulations 
at $300 \mathrm{~K}$, and the dynamics of CV1 at the air-water interface are shown in Figure 8a. The dynamical behavior of CV1 suggests that all the available reactant and product regions are sufficiently explored, and the free energy profile is converged. In this well-tempered metadynamics simulation, the deprotonation of acetic acid at the air-water interface proceeds from product to reactant via a single transition state, as shown in Figure 8b. Two well-defined minima can be observed in the free energy profile. The minimum at 0.90 corresponds to the un-dissociated neutral acetic acid, and the minimum at 0.20 refers to the dissociated acetate anion. The transition state barrier for the deprotonation (labeled as TS in the free energy profile) is located between the $\mathrm{R}$ and $\mathrm{P}$ states. The CV1 value for the transition state is 0.66 , which corresponds to a free energy barrier of $20.30 \mathrm{~kJ} / \mathrm{mol}$ for acetic acid deprotonation at the air-water interface. The reactant $(\mathrm{R})$, transition state $(\mathrm{TS})$, and product $(\mathrm{P})$ are shown in Figure 8c. In the reactant state, the hydroxyl group of acetic acid is hydrogen-bonded to a water molecule. In the transition state, the hydroxyl group of acetic acid is still hydrogenbonded to the water molecule, but the bond length in the hydroxyl O-H group is stretched. In the product state, the hydroxyl O-H group in the acetic acid molecule is broken, and the proton is shifted to a neighboring water molecule, forming a hydronium $\left(\mathrm{H}_{3} \mathrm{O}^{+}\right)$ion. Figures S10-S11 in the Supplementary Material give a detailed analysis and confirmation of the free energy convergence between the transition state and product basin. We have performed additional metadynamics calculations (using different initial conditions) for the air-water interface. The fluctuations of CV1 values and free energy profiles are shown in Figure S12 in the Supplementary Material. In this case, the reactant goes to the product state via a single transition state with a free energy barrier value of $21.03 \mathrm{~kJ} / \mathrm{mol}$. The average free energy barrier value obtained from the different initial conditions is $20.66 \mathrm{~kJ} / \mathrm{mol}$. A $7.5 \mathrm{~kJ} / \mathrm{mol}$ free energy barrier was previously reported for DFT-based metadynamics simulations of formic acid (a similar carboxylic acid). ${ }^{31}$ As such, our computed free energy barrier for the acetic acid deprotonation is higher than that of formic acid at the air-water interface. The various distances between the carboxylate oxygen and neighboring hydrogen atoms of water 
molecules at the air-water interface are shown in Figure S13 of the Supplementary Material. These calculations suggest that re-protonation does not occur after acetic deprotonation at the air-water interface within the time scales probed in our simulations.

The slightly lower free energy barrier for the slab configuration indicates that acetic acid deprotonates faster at an air-water interface than in a bulk aqueous environment. A comprehensive analysis of the solvation shell structure and hydrogen bond dynamics of acetic acid-water in the bulk aqueous environment and at the interface is less clear since almost the same average number of hydrogen bonds are formed by the hydroxyl group. Previous studies on the deprotonation of formic ${ }^{31}$ and carbonic acid ${ }^{68}$ at the air-water interface proposed that faster deprotonation may be due to faster water re-orientation/hydrogen bond dynamics or second-solvation shell effects. To precisely assess these effects, we carried out RDF calculations between the hydroxyl oxygen of acetic acid and the oxygen atom of the surrounding water molecules. Figure S14 shows the $\mathrm{O}_{-} \mathrm{O}_{w}$ RDF calculations in the bulk aqueous environment and at the air-water interface in the Supplementary Material. The $\mathrm{O}-\mathrm{O}_{w} \mathrm{RDF}$ at the air-water interface indicates that the water molecules are well structured around the hydroxyl (O-H) group, even up to the third solvation shell. This is in stark contrast with the $\mathrm{O}-\mathrm{O}_{w}$ RDF in the bulk aqueous environment, where second and third solvation shells are not well structured (i.e., water molecules exceeding the first solvation shell are randomly arranged). Therefore, we suggest that the effect of the solvation shell could be one reason for faster deprotonation at the air-water interface. Recently, surface-sensitive measurements using online electrospray ionization mass spectrometry (OESI-MS) of acetic acid and pyruvic acids suggested enhanced acidity of these carboxylic acids on the surface of water. ${ }^{18}$ Francisco and co-workers further reported that the $p K_{a}$ value and redox potential at the air-water interface differed from a bulk aqueous environment. ${ }^{69,70}$ Their study also inferred that the air-water interface provides an energetically favorable environment for redox reactions. All of these prior studies collectively demonstrate that the enhancement of acidities could be another reason for the lower small free energy barrier for acetic acid 
deprotonation at the air-water interface, which supports our metadynamics simulations.

The behavior of acetic acid at the air-water interface is qualitatively different than the dynamics of strong acids. For example, hydrochloric $(\mathrm{HCl})$ acid has a small free energy barrier for the deprotonation at the interface as in the bulk. ${ }^{71}$ On the other hand, nitric acid $\left(\mathrm{HNO}_{3}\right)$ has an exceptionally high free energy barrier of deprotonation at the interface due to an inadequate solvation shell and its extraordinary stability. ${ }^{71-73}$ However, all the carboxylic acids have a strong surface propensity: the faster deprotonation at the interface could indicate that the role of these weak acids in acidifying the polluted atmosphere, water droplets, rain, and cloud water may be more critical than initially thought.

\section{Conclusions}

In this study, we have carried out extensive ab initio molecular dynamics simulations of acetic acid to understand and contrast its dynamical effects in a bulk aqueous vs. an airwater interface environment. Our calculations demonstrate that acetic acid is particularly surface-active and remains near the air-water interface at all temperatures. Furthermore, our hydrogen-bond analysis indicates that the $\mathrm{H} \cdots \mathrm{O}_{w}$ hydrogen bond is more dominant than the $\mathrm{O} \cdots \mathrm{H}_{w}$ hydrogen bond between acetic acid and neighboring water molecules, which arises due to the higher particle density of water molecules around the hydroxyl $(\mathrm{OH})$ group. Our calculations suggest a negligible difference in the hydrogen bond lifetime of the $\mathrm{O} \cdot \mathrm{H}_{w}$ pair in either the bulk aqueous environment or at the air-water interface at $300 \mathrm{~K}$. We also observed a faster decay of the $\mathrm{O} \cdot \mathrm{H}_{w}$ hydrogen-bond pair as a function of temperature at the interface. However, a slower decay of the $\mathrm{H} \cdots \mathrm{O}_{w}$ pair was observed in the bulk aqueous environment compared to the air-water interface due to a higher particle density. Furthermore, deprotonation of acetic acid in either the bulk or interfacial environments was not observed. Our ab initio metadynamics simulations provide an atomistic-level picture of the reaction path, as well as numerical predictions of the energetic landscape in both bulk 
and air-water environments. Using ab initio-based metadynamics to predict the free energy profile of the deprotonation process, we have shown that the surface free energy barrier is lower than its bulk value. Our findings suggest that this reduction of the free energy barrier at the air-water interface is due to a well-structured solvation shell. Collectively, our metadynamics calculations (1) show that acetic acid deprotonation occurs more readily on an aqueous surface than in a bulk water environment and (2) provide additional, critical mechanistic insight into acid-base reactions at the air-water interface.

\section{Acknowledgement}

This work was supported by the U.S. Department of Energy, Office of Science, Early Career Research Program under Award No. DE-SC0016269.

\section{Supporting Information Available}

Additional materials on the hydroxyl bond length in acetic acid, combined distribution functions (CDFs), additional free energy profiles, convergence tests of the metadynamics calculations, and radial distribution functions.

\section{References}

(1) Mishra, H.; Enami, S.; Nielsen, R. J.; Stewart, L. A.; Hoffmann, M. R.; Goddard, W. A.; Colussi, A. J. Brønsted basicity of the air-water interface. Proc. Nat. Acad. Sci. USA 2012, 109, 18679-18683.

(2) Parashar, S.; Lesnicki, D.; Sulpizi, M. Increased Acid Dissociation at the Quartz/Water Interface. J. Phys. Chem. Lett. 2018, 9, 2186-2189. 
(3) Yano, Y. F. Kinetics of protein unfolding at interfaces. J. Phys. Condens. Matter 2012, 24, 503101 .

(4) Finlayson-Pitts, B. J. Reactions at surfaces in the atmosphere: integration of experiments and theory as necessary (but not necessarily sufficient) for predicting the physical chemistry of aerosols. Phys. Chem. Chem. Phys. 2009, 11, 7760-7779.

(5) Jubb, A. M.; Allen, H. C. Bisulfate Dehydration at Air/Solution Interfaces Probed by Vibrational Sum Frequency Generation Spectroscopy. J. Phys. Chem. C 2012, 116, $13161-13168$.

(6) Hub, J. S.; Wolf, M. G.; Caleman, C.; van Maaren, P. J.; Groenhof, G.; van der Spoel, D. Thermodynamics of hydronium and hydroxide surface solvation. Chem. Sci. 2014, 5, $1745-1749$.

(7) Strazdaite, S.; Meister, K.; Bakker, H. J. Reduced Acid Dissociation of Amino-Acids at the Surface of Water. J. Am. Chem. Soc. 2017, 139, 3716-3720.

(8) Finlayson-Pitts, B.; James Pitts, J. Chemistry of the Upper and Lower Atmosphere: Theory, Experiments, and Applications, 1st ed.; Academic Press: San Diego, CA, USA, 1999.

(9) Wayne, R. P. An Introduction to the Chemistry of the Atmospheres of Earth, the Planets, and their Satellites, 3rd ed.; Oxford University Press: Oxford, 2000.

(10) Chanda, S. In Encyclopedia of Toxicology (Second Edition), second edition ed.; Wexler, P., Ed.; Elsevier: New York, 2005; pp $25-26$.

(11) Schobesberger, S.; Lopez-Hilfiker, F. D.; Taipale, D.; Millet, D. B.; D'Ambro, E. L.; Rantala, P.; Mammarella, I.; Zhou, P.; Wolfe, G. M.; Lee, B. H.; Boy, M.; Thornton, J. A. High upward fluxes of formic acid from a boreal forest canopy. Geophys. Res. Lett. 2016, 43, 9342-9351. 
(12) Munger, J. W.; Jr., J. C.; Jr., B. C. D.; Hoffmann, M. R. Carboxylic acids and carbonyl compounds in southern California clouds and fogs. Tellus B: Chemical and Physical Meteorology 1989, 41, 230-242.

(13) Weathers, K. C.; Likens, G. E.; Bormann, F. H.; Bicknell, S. H.; Bormann, B. T.; Daube, B. C.; Eaton, J. S.; Galloway, J. N.; Keene, W. C.; et al., . Cloudwater chemistry from ten sites in North America. Environ. Sci. Technol. 1988, 22, 1018-1026.

(14) Durana, N.; Casado, H.; García, C.; Ezcurra, A.; Lacaux, J.; Encinas, D. Organic acids in precipitation in the Basque country (North of Spain). Atmos. Res. 1992, 28, 93 101.

(15) Puxbaum, H.; Rosenberg, C.; Gregori, M.; Lanzerstorfer, C.; Ober, E.; Winiwarter, W. Atmospheric concentrations of formic and acetic acid and related compounds in eastern and northern Austria. Atmos. Environ. (1967) 1988, 22, 2841 - 2850.

(16) Khare, P.; Kumar, N.; Kumari, K. M.; Srivastava, S. S. Atmospheric formic and acetic acids: An overview. Rev. Geophys. 1999, 37, 227-248.

(17) Keene, W. C.; Galloway, J. N. Organic acidity in precipitation of North America. Atmos. Environ. (1967) 1984, 18, $2491-2497$.

(18) Eugene, A. J.; Pillar, E. A.; Colussi, A. J.; Guzman, M. I. Enhanced Acidity of Acetic and Pyruvic Acids on the Surface of Water. Langmuir 2018, 34, 9307-9313, PMID: 29975541.

(19) MacInnes, D. A.; Shedlovsky, T. The Determination of the Ionization Constant of Acetic Acid, at $25^{\circ}$, from Conductance Measurements. J. Am. Chem. Soc. 1932, 54 , $1429-1438$.

(20) Génin, F.; Quilès, F.; Burneau, A. Infrared and Raman spectroscopic study of carboxylic acids in heavy water. Phys. Chem. Chem. Phys. 2001, 3, 932-942. 
(21) Brown, M. A.; Vila, F.; Sterrer, M.; Thürmer, S.; Winter, B.; Ammann, M.; Rehr, J. J.; van Bokhoven, J. A. Electronic Structures of Formic Acid (HCOOH) and Formate $\left(\mathrm{HCOO}^{-}\right)$in Aqueous Solutions. J. Phys. Chem. Lett. 2012, 3, 1754-1759.

(22) Pruyne, J. G.; Lee, M.-T.; Fábri, C.; Beloqui Redondo, A.; Kleibert, A.; Ammann, M.; Brown, M. A.; Krisch, M. J. Liquid-Vapor Interface of Formic Acid Solutions in Salt Water: A Comparison of Macroscopic Surface Tension and Microscopic in Situ X-ray Photoelectron Spectroscopy Measurements. J. Phys. Chem. C 2014, 118, 29350-29360.

(23) Ottosson, N.; Wernersson, E.; Söderström, J.; Pokapanich, W.; Kaufmann, S.; Svensson, S.; Persson, I.; Öhrwall, G.; Björneholm, O. The protonation state of small carboxylic acids at the water surface from photoelectron spectroscopy. Phys. Chem. Chem. Phys. 2011, 13, 12261-12267.

(24) Johnson, C. M.; Tyrode, E.; Kumpulainen, A.; Leygraf, C. Vibrational Sum Frequency Spectroscopy Study of the Liquid/Vapor Interface of Formic Acid/Water Solutions. J. Phys. Chem. C 2009, 113, 13209-13218.

(25) Johnson, C. M.; Baldelli, S. Vibrational Sum Frequency Spectroscopy Studies of the Influence of Solutes and Phospholipids at Vapor/Water Interfaces Relevant to Biological and Environmental Systems. Chem. Rev. 2014, 114, 8416-8446.

(26) Pu, L.; Sun, Y.; Zhang, Z. Hydrogen Bonding of Hydrates of Double Acetic Acid Molecules. J. Phys. Chem. A 2009, 113, 6841-6848.

(27) Krishnakumar, P.; Maity, D. K. Theoretical studies on dimerization vs. microhydration of carboxylic acids. Comput. Theor. Chem. 2017, 1099, 185 - 194.

(28) Park, J. M.; Laio, A.; Iannuzzi, M.; Parrinello, M. Dissociation Mechanism of Acetic Acid in Water. J. Am. Chem. Soc. 2006, 128, 11318-11319. 
(29) Nishida, N.; Tokushima, T.; Takahashi, O. A theoretical study on the pH dependence of X-ray emission spectra for aqueous acetic acid. Chemical Physics Letters 2016, 649, $156-161$.

(30) Compoint, M.; Toubin, C.; Picaud, S.; Hoang, P.; Girardet, C. Geometry and dynamics of formic and acetic acids adsorbed on ice. Chemical Physics Letters 2002, 365, 1 - 7 .

(31) Murdachaew, G.; Nathanson, G. M.; Benny Gerber, R.; Halonen, L. Deprotonation of formic acid in collisions with a liquid water surface studied by molecular dynamics and metadynamics simulations. Phys. Chem. Chem. Phys. 2016, 18, 29756-29770.

(32) VandeVondele, J.; Hutter, J. An efficient orbital transformation method for electronic structure calculations. J. Chem. Phys. 2003, 118, 4365-4369.

(33) VandeVondele, J.; Krack, M.; Mohamed, F.; Parrinello, M.; Chassaing, T.; Hutter, J. QUICKSTEP: Fast and accurate density functional calculations using a mixed Gaussian and plane waves approach. Comput. Phys. Commun. 2005, 167, 103-128.

(34) Krack, M.; Parrinello, M. All-electron ab-initio molecular dynamics. Phys. Chem. Chem. Phys. 2000, 2, 2105-2112.

(35) Hutter, J.; Iannuzzi, M.; Schiffmann, F.; VandeVondele, J. cp2k: atomistic simulations of condensed matter systems. Wiley Interdiscip. Rev. Comput. Mol. Sci. 2014, 4, 15-25.

(36) https://www.cp2k.org.

(37) Lippert, G.; Hutter, J.; Parrinello, M. A hybrid Gaussian and plane wave density functional scheme. Mol. Phys. 1997, 92, 477-488.

(38) Goedecker, S.; Teter, M.; Hutter, J. Separable dual-space Gaussian pseudopotentials. Phys. Rev. B 1996, 54, 1703-1710.

(39) Hartwigsen, C.; Goedecker, S.; Hutter, J. Relativistic separable dual-space Gaussian pseudopotentials from H to Rn. Phys. Rev. B 1998, 58, 3641-3662. 
(40) Schäfer, A.; Huber, C.; Ahlrichs, R. Fully optimized contracted Gaussian basis sets of triple zeta valence quality for atoms Li to Kr. J. Chem. Phys. 1994, 100, 5829-5835.

(41) Becke, A. D. Density-functional thermochemistry. III. The role of exact exchange. J. Chem. Phys. 1993, 98, 5648-5652.

(42) Becke, A. D. Density-functional exchange-energy approximation with correct asymptotic behavior. Phys. Rev. A 1988, 38, 3098-3100.

(43) Lee, C.; Yang, W.; Parr, R. G. Development of the Colle-Salvetti correlation-energy formula into a functional of the electron density. Phys. Rev. B 1988, 37, 785-789.

(44) Grimme, S. Semiempirical GGA-type density functional constructed with a long-range dispersion correction. J. Comput. Chem. 2006, 27, 1787-1799.

(45) Kolafa, J. Time-reversible always stable predictor-corrector method for molecular dynamics of polarizable molecules. J. Comput. Chem. 2004, 25, 335-342.

(46) Kühne, T. D.; Krack, M.; Mohamed, F. R.; Parrinello, M. Efficient and Accurate CarParrinello-like Approach to Born-Oppenheimer Molecular Dynamics. Phys. Rev. Lett. 2007, 98, 066401.

(47) Nosé, S. A unified formulation of the constant temperature molecular dynamics methods. J. Chem. Phys. 1984, 81, 511-519.

(48) Hoover, W. G. Canonical dynamics: Equilibrium phase-space distributions. Phys. Rev. A 1985, 31, 1695-1697.

(49) Martínez, L.; Andrade, R.; Birgin, E. G.; Martínez, J. M. PACKMOL: A package for building initial configurations for molecular dynamics simulations. J. Comput. Chem. 2009, 30, 2157-2164.

(50) Schmidt, J.; VandeVondele, J.; Kuo, I.-F. W.; Sebastiani, D.; Siepmann, J. I.; Hutter, J.; Mundy, C. J. Isobaric-Isothermal Molecular Dynamics Simulations Utilizing Density 
Functional Theory: An Assessment of the Structure and Density of Water at NearAmbient Conditions. J. Phys. Chem. B 2009, 113, 11959-11964.

(51) Murdachaew, G.; Mundy, C. J.; Schenter, G. K.; Laino, T.; Hutter, J. Semiempirical Self-Consistent Polarization Description of Bulk Water, the Liquid-Vapor Interface, and Cubic Ice. J. Phys. Chem. A 2011, 115, 6046-6053.

(52) Hammerich, A. D.; Finlayson-Pitts, B. J.; Gerber, R. B. Mechanism for formation of atmospheric $\mathrm{Cl}$ atom precursors in the reaction of dinitrogen oxides with $\mathrm{HCl} / \mathrm{Cl}^{-}$on aqueous films. Phys. Chem. Chem. Phys. 2015, 17, 19360-19370.

(53) Partanen, L.; Murdachaew, G.; Gerber, R. B.; Halonen, L. Temperature and collision energy effects on dissociation of hydrochloric acid on water surfaces. Phys. Chem. Chem. Phys. 2016, 18, 13432-13442.

(54) Biswas, S.; Kwon, H.; Barsanti, K. C.; Myllys, N.; Smith, J. N.; Wong, B. M. Ab initio metadynamics calculations of dimethylamine for probing $\mathrm{pK}_{b}$ variations in bulk vs. surface environments. Phys. Chem. Chem. Phys. 2020, 22, 26265-26277.

(55) Kuo, I.-F. W.; Mundy, C. J. An ab Initio Molecular Dynamics Study of the Aqueous Liquid-Vapor Interface. Science 2004, 303, 658-660.

(56) Dodia, M.; Ohto, T.; Imoto, S.; Nagata, Y. Structure and Dynamics of Water at the Water-Air Interface Using First-Principles Molecular Dynamics Simulations. II. NonLocal vs Empirical van der Waals Corrections. J. Chem. Theory Comput. 2019, 15, $3836-3843$.

(57) Del Ben, M.; Hutter, J.; VandeVondele, J. Probing the structural and dynamical properties of liquid water with models including non-local electron correlation. J. Chem. Phys. 2015, 143, 054506. 
(58) Halstead, S. J. A molecular dynamics investigation of the dissociation constants of acetic and oxalic acid in supercritical water. Mol. Phys. 2016, 114, 1915-1921.

(59) Luzar, A.; Chandler, D. Hydrogen-bond kinetics in liquid water. Nature 1996, 379, $55-57$.

(60) Luzar, A.; Chandler, D. Effect of Environment on Hydrogen Bond Dynamics in Liquid Water. Phys. Rev. Lett. 1996, 76, 928-931.

(61) Luzar, A. Resolving the hydrogen bond dynamics conundrum. J. Chem. Phys. 2000, 113, 10663-10675.

(62) Xu, H.; Berne, B. J. Hydrogen-Bond Kinetics in the Solvation Shell of a Polypeptide. J. Phys. Chem. B 2001, 105, 11929-11932.

(63) Brehm, M.; Kirchner, B. TRAVIS - A Free Analyzer and Visualizer for Monte Carlo and Molecular Dynamics Trajectories. J. Chem. Inf. Model. 2011, 51, 2007-2023.

(64) Brehm, M.; Thomas, M.; Gehrke, S.; Kirchner, B. TRAVIS-A free analyzer for trajectories from molecular simulation. J. Chem. Phys. 2020, 152, 164105.

(65) Tummanapelli, A. K.; Vasudevan, S. Dissociation Constants of Weak Acids from ab Initio Molecular Dynamics Using Metadynamics: Influence of the Inductive Effect and Hydrogen Bonding on $\mathrm{pK}_{a}$ Values. J. Phys. Chem. B 2014, 118, 13651-13657.

(66) Daub, C. D.; Halonen, L. Ab Initio Molecular Dynamics Simulations of the Influence of Lithium Bromide Salt on the Deprotonation of Formic Acid in Aqueous Solution. J. Phys. Chem. B 2019, 123, 6823-6829.

(67) Lee, J.-G.; Asciutto, E.; Babin, V.; Sagui, C.; Darden, T.; Roland, C. Deprotonation of Solvated Formic Acid: Car-Parrinello and Metadynamics Simulations. J. Phys. Chem. B 2006, 110, 2325-2331. 
(68) Galib, M.; Hanna, G. Molecular dynamics simulations predict an accelerated dissociation of $\mathrm{H} 2 \mathrm{CO} 3$ at the air-water interface. Phys. Chem. Chem. Phys. 2014, 16, $25573-25582$.

(69) Zhu, C.; Kumar, M.; Zhong, J.; Li, L.; Francisco, J. S.; Zeng, X. C. New Mechanistic Pathways for Criegee-Water Chemistry at the Air/Water Interface. J. Am. Chem. Soc. 2016, 138, 11164-11169.

(70) Zhong, J.; Kumar, M.; Francisco, J. S.; Zeng, X. C. Insight into Chemistry on Cloud/Aerosol Water Surfaces. Acc. Chem. Res. 2018, 51, 1229-1237.

(71) Baer, M. D.; Tobias, D. J.; Mundy, C. J. Investigation of Interfacial and Bulk Dissociation of $\mathrm{HBr}, \mathrm{HCl}$, and $\mathrm{HNO}_{3}$ Using Density Functional Theory-Based Molecular Dynamics Simulations. J. Phys. Chem. C 2014, 118, 29412-29420.

(72) Shamay, E. S.; Buch, V.; Parrinello, M.; Richmond, G. L. At the Water's Edge: Nitric Acid as a Weak Acid. J. Am. Chem. Soc. 2007, 129, 12910-12911.

(73) Wang, S.; Bianco, R.; Hynes, J. T. Depth-Dependent Dissociation of Nitric Acid at an Aqueous Surface: Car-Parrinello Molecular Dynamics. J. Phys. Chem. A 2009, 113, $1295-1307$. 


\section{${ }_{635}$ TOC GRAPHIC}

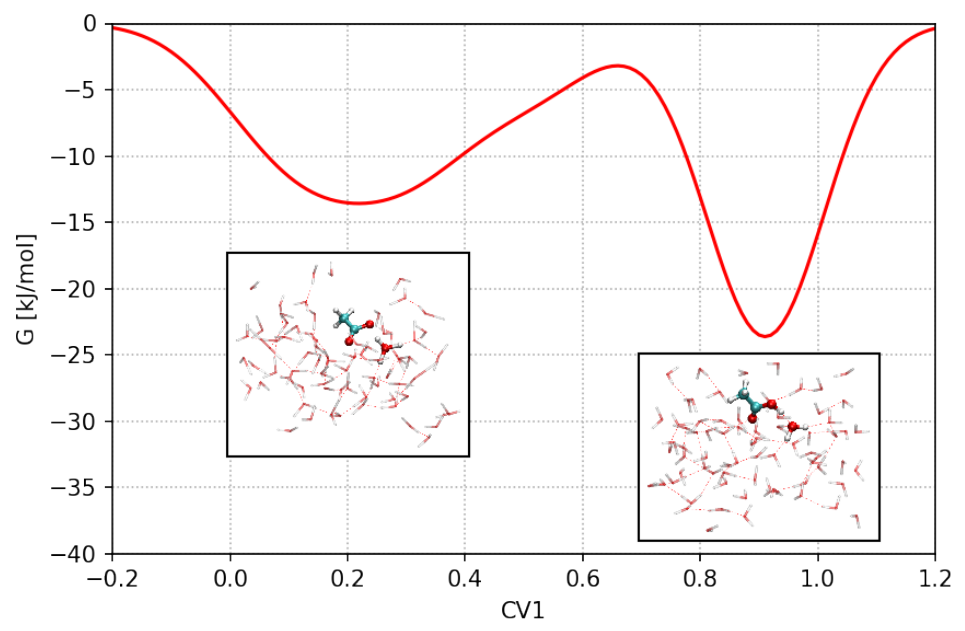

636 